\title{
GLASGOW UNIVERSITY RADIOCARBON MEASUREMENTS VI
}

\author{
J. G. FARMER, M. J. STENHOUSE, and M. S. BAXTER \\ Chemistry Department, The University, Glasgow, G12 8QQ
}

\section{INTRODUCTION}

The following list presents results obtained during 1970-1971 and is a continuation of research of which data have been pub. previously (Baxter et al., 1969; Ergin et al., 1970; Baxter and Walton, 1970; Harkness and Walton, 1972; Ergin et al., 1972). The major research efforts at Glasgow are aimed at evaluation of (a) natural annual $\mathrm{C}^{14}$ levels and (b) burdens and residence times of artificial $\mathrm{C}^{14}$ in the environment and human tissues. The results of these studies are presented as $\delta \mathrm{C}^{14}$ and $\Delta$ values based on age-corrected activities, although this correction is very small. The errors quoted are counting uncertainties only, expressed at the $1 \sigma$ level. Pretreatment procedures are outlined in the text and analytical methods are essentially unchanged. Gas proportional counting of both $\mathrm{CO}_{2}$ and $\mathrm{CH}_{4}$ is employed in $2.6 \mathrm{~L}$ and $0.5 \mathrm{~L}$ detectors, respectively. Mass spectrometric analyses are performed to a precision of $0.1 \% o( \pm 2 \sigma)$ on a V.G. Micromass 602B stable isotope mass spectrometer.

\section{ACKNOWLEDGMENTS}

This work is supported by generous grants from the Natural Environment Research Council and the Medical Research Council. We wish to thank the many individuals who contributed to the supply of sample materials. We are also grateful to T. D. B. Lyon for development of mass spectrometric techniques and to J. Brown for efficient technical assistance.

\section{SAMPLE DESCRIPTIONS}

\section{TREE-RING SAMPLES}

A study of 19th century atmospheric $\mathrm{C}^{14}$ concentrations has begun through analyses of annual rings of Oak (Quercus robur) planted in 1810 (felled 1970) in Russell's Enclosure, Forest of Dean $\left(51^{\circ} 48^{\prime} \mathrm{N}\right.$ Lat, $2^{\circ}$ $37^{\prime}$ W Long), Gloucestershire, England. Rings id. by calendar yr by F. S. Walker with J. M. Fletcher, Research Lab. for Archaeol. and History of Art, Oxford. Wood of each ring was split into thin shavings, extracted with $40 / 60$ petroleum ether, and bleached to yield the pure cellulose fraction. The cellulose was charred at $500^{\circ} \mathrm{C}$ prior to combustion in the gas preparation system. 
Tree rings, Forest of Dean series

\begin{tabular}{|c|c|c|c|c|}
\hline Sample no. & $\mathrm{Yr}$ & $\delta \mathrm{C}^{14 \%} \%$ & $\delta \mathrm{C}^{130} \%$ & $\Delta \%$ \\
\hline GU-381 & 1829 & $+2.0 \pm 0.6$ & -23.4 & $+1.7 \pm 0.6$ \\
\hline GU-382 & 1830 & $+0.9 \pm 0.5$ & -23.6 & $+0.6 \pm 0.5$ \\
\hline GU-383 & 1831 & $+1.4 \pm 0.6$ & -24.1 & $+1.3 \pm 0.6$ \\
\hline GU-384 & 1832 & $+0.1 \pm 0.6$ & -24.2 & $-0.1 \pm 0.6$ \\
\hline GU-385 & 1833 & $+0.8 \pm 0.5$ & -24.3 & $+0.6 \pm 0.5$ \\
\hline GU-386 & 1834 & $-0.3 \pm 0.8$ & -25.0 & $-0.3 \pm 0.8$ \\
\hline GU-387 & 1836 & $+2.0 \pm 0.6$ & -23.6 & $+1.7 \pm 0.6$ \\
\hline GU-388 & 1838 & $+1.2 \pm 0.5$ & -24.8 & $+1.2 \pm 0.5$ \\
\hline GU-389 & 1839 & $+0.2 \pm 0.6$ & -24.7 & $+0.1 \pm 0.6$ \\
\hline GU-390 & 1840 & $+0.7 \pm 0.5$ & -23.9 & $+0.5 \pm 0.5$ \\
\hline GU-391 & 1841 & $+0.6 \pm 0.6$ & -24.0 & $+0.4 \pm 0.6$ \\
\hline GU-392 & 1842 & $-0.3 \pm 0.6$ & -24.3 & $-0.5 \pm 0.6$ \\
\hline GU-393 & 1843 & $-0.7 \pm 0.6$ & -24.6 & $-0.8 \pm 0.6$ \\
\hline GU-394 & 1844 & $-0.3 \pm 0.5$ & -24.2 & $-0.5 \pm 0.5$ \\
\hline GU-395 & 1849 & $+0.2 \pm 0.5$ & -23.4 & $-0.2 \pm 0.5$ \\
\hline GU-396 & 1850 & $+0.2 \pm 0.6$ & -24.6 & $+0.1 \pm 0.6$ \\
\hline GU-397 & 1851 & $-0.3 \pm 0.6$ & -25.0 & $-0.3 \pm 0.6$ \\
\hline GU-398 & 1852 & $-2.0 \pm 0.6$ & -23.9 & $-2.2 \pm 0.6$ \\
\hline GU-399 & 1853 & $-2.8 \pm 0.6$ & -25.3 & $-2.7 \pm 0.6$ \\
\hline GU-400 & 1854 & $-0.7 \pm 0.6$ & -23.3 & $-1.0 \pm 0.6$ \\
\hline GU-401 & 1855 & $-0.5 \pm 0.6$ & -23.3 & $-0.8 \pm 0.6$ \\
\hline GU-402 & 1856 & $-1.4 \pm 0.5$ & -23.9 & $-1.6 \pm 0.5$ \\
\hline GU-403 & 1857 & $-0.5 \pm 0.6$ & -24.5 & $-0.6 \pm 0.6$ \\
\hline GU-404 & 1858 & $+1.5 \pm 0.6$ & -24.2 & $+1.3 \pm 0.6$ \\
\hline GU-405 & 1859 & $-0.3 \pm 0.5$ & -24.2 & $-0.4 \pm 0.5$ \\
\hline GU-406 & 1860 & $-0.2 \pm 0.5$ & -24.5 & $-0.3 \pm 0.5$ \\
\hline GU-407 & 1861 & $-0.1 \pm 0.6$ & -24.4 & $-0.2 \pm 0.6$ \\
\hline GU-408 & 1862 & $+0.3 \pm 0.6$ & -24.4 & $+0.2 \pm 0.6$ \\
\hline GU-409 & 1863 & $-1.3 \pm 0.5$ & -23.4 & $-1.6 \pm 0.5$ \\
\hline GU-410 & 1864 & $-2.0 \pm 0.6$ & -22.9 & $-2.4 \pm 0.6$ \\
\hline GU-411 & 1865 & $-0.6 \pm 0.6$ & -23.8 & $-0.8 \pm 0.6$ \\
\hline
\end{tabular}

Comment: these results suggest $\mathrm{N}$ hemisphere $\mathrm{C}^{14}$ activities fluctuated significantly on an annual basis from 1829 to 1865 . Baxter and Walton (1971) previously reported significant annual variations for period 1890 to 1950 in $\mathrm{N}$ hemisphere.

\section{VINTAGE WINE SAMPLES}

The study of atmospheric $\mathrm{C}^{14}$ concentrations in past years through analyses of vintage wine samples (Baxter and Walton, 1970) was extended to $S$ hemisphere. Several wine samples from Australia were analyzed. The grapes were picked in March of the year of production of each wine sample, after ca. 4 to 5 mos. on the vine. 
Vintage wine, Australia series

\begin{tabular}{lcccc}
\hline Sample no. & $\mathrm{Yr}$ & $\delta \mathrm{C}^{14 \%} \%$ & $\delta \mathrm{C}^{13 \%} \%$ & $\Delta \%$ \\
\hline GU-412 & 1919 & $-2.6 \pm 0.5$ & -25.6 & $-2.5 \pm 0.5$ \\
GU-413 & 1928 & $-1.6 \pm 0.6$ & -26.4 & $-1.3 \pm 0.6$ \\
GU-414 & 1933 & $-1.2 \pm 0.5$ & -29.6 & $-0.3 \pm 0.5$ \\
GU-415 & 1934 & $-2.8 \pm 0.5$ & -27.4 & $-2.3 \pm 0.5$ \\
GU-416 & 1936 & $-2.7 \pm 0.5$ & -27.4 & $-2.2 \pm 0.5$ \\
GU-417 & 1940 & $-3.5 \pm 0.6$ & -26.9 & $-3.2 \pm 0.6$ \\
GU-418 & 1944 & $-1.8 \pm 0.5$ & -25.5 & $-1.7 \pm 0.5$ \\
\hline
\end{tabular}

Comment: further measurements are being made on wheat seeds, oats, wines, and annual tree rings. Correction for industrial Suess effect will enable assessment of natural $\mathrm{C}^{14}$ fluctuations.

\section{HUMAN TISSUE SAMPLES}

The $\mathrm{C}^{14}$ activity in individual human tissues is being measured in specimens of varying age at time of death. Human tissue $\mathrm{C}^{14}$ measurements have already been obtained at this lab. (Harkness and Walton, 1972); the present study is a continuation of previous work using similar pretreatment and analytical procedures. Tissues were first washed with distilled water to remove external traces of blood, separated from fat, and homogenized; 15 to $25 \mathrm{mls}$ distilled water were added, if necessary, before homogenizing. The resulting solution was freeze-dried and the product material combusted; $20 \mathrm{~g}$ of tissue yielded ca. $2 \mathrm{~g}$ to $5 \mathrm{~g}$ of dried material, the amount depending on the particular tissue. Bone samples were first cut into $<1 \mathrm{~cm}^{3}$ pieces; fragments were boiled in $2 \mathrm{M} \mathrm{NH} \mathrm{NH}_{4} \mathrm{OH}$, which released marrow fat into the aqueous phase. Marrow fat was isolated by benzene extraction followed by solvent distillation. The remaining bone sample was digested in $2 \mathrm{M} \mathrm{HCl}$, during which, hydrolysis of the protein (collagen) fraction occurred. Collagen was obtained by evaporating the solution to dryness so that collagen was absorbed on the calcium phosphate residue which was combusted.

\section{0-yr-old human tissues, series}

Samples GU-419 to -423 from a 50-yr-old male who died Jan. 10, 1971 of cerebral haemorrhage.

\begin{tabular}{clccc}
\hline Sample no. & Tissue & $\delta \mathrm{C}^{14 \%}$ & $\delta \mathrm{C}^{13 \%} \%$ & $\Delta \%$ \\
\hline GU-419 & Liver & $44.3 \pm 0.8$ & -21.9 & $43.4 \pm 0.8$ \\
$*$ GU-420 & Muscle & $51.0 \pm 1.2$ & -18.2 & $48.9 \pm 1.2$ \\
GU-421 & Testes & $38.3 \pm 1.0$ & -23.4 & $37.8 \pm 1.0$ \\
*GU-422 & Heart & $53.3 \pm 1.8$ & -4.1 & $46.9 \pm 1.8$ \\
GU-423 & Kidney & $45.1 \pm 0.8$ & -24.0 & $44.8 \pm 0.8$ \\
\hline
\end{tabular}

* Samples diluted with inactive $\mathrm{CO}_{2}$. 


\section{2-yr-old human tissues, series}

Samples GU-424 to -434 from a 72-yr-old male who died March 24, 1971 of bronchial pneumonia.

\begin{tabular}{llrrr}
\hline Sample no. & Tissue & $\delta \mathrm{C}^{14 \%} \%$ & $\delta \mathrm{C}^{13 \%} \%$ & \multicolumn{1}{c}{$\Delta \%$} \\
\hline GU-424 & Brain & $47.0 \pm 0.8$ & -21.5 & $46.0 \pm 0.8$ \\
GU-425 & Muscle & $47.3 \pm 0.9$ & -24.2 & $47.1 \pm 1.0$ \\
GU-426 & Pancreas & $49.8 \pm 0.8$ & -26.0 & $50.1 \pm 0.8$ \\
GU-427 & Lung & $47.4 \pm 0.9$ & -22.7 & $46.7 \pm 0.9$ \\
GU-428 & Spleen & $47.8 \pm 0.9$ & -22.1 & $46.9 \pm 1.0$ \\
GU-429 & Liver & $49.4 \pm 0.9$ & -22.5 & $48.7 \pm 0.9$ \\
GU-430 & Heart & $47.1 \pm 0.9$ & -21.8 & $46.2 \pm 0.9$ \\
GU-431 & Kidney & $50.9 \pm 0.8$ & -24.1 & $50.6 \pm 0.8$ \\
GU-432 & Testes & $45.1 \pm 0.9$ & -24.6 & $45.0 \pm 0.9$ \\
GU-433 & Marrow fat & $47.7 \pm 0.9$ & -27.1 & $48.3 \pm 1.0$ \\
*GU-434 & Collagen & $8.2 \pm 2.7$ & -7.9 & $4.5 \pm 2.9$
\end{tabular}

* Sample diluted with inactive $\mathrm{CO}_{2}$.

\section{4-yr-old human tissues, series}

Samples GU-435 to -440 from a 64-yr-old female who died June 2, 1971 of pulmonary embolism.

\begin{tabular}{llccc}
\hline Sample no. & Tissue & $\delta \mathrm{C}^{14} \%$ & $\delta \mathrm{C}^{13 \%} \%$ & $\Delta \%$ \\
\hline GU-435 & Muscle & $47.9 \pm 0.9$ & -24.0 & $47.6 \pm 1.0$ \\
GU-436 & Brain & $47.3 \pm 0.9$ & -24.9 & $47.3 \pm 0.9$ \\
GU-437 & Fat & $45.7 \pm 1.2$ & -25.0 & $45.7 \pm 1.3$ \\
GU-438 & Heart & $41.7 \pm 0.9$ & -22.2 & $40.9 \pm 0.9$ \\
GU-439 & Kidney & $48.9 \pm 0.9$ & -22.5 & $48.2 \pm 1.0$ \\
*GU-440 & Ovaries & $46.0 \pm 1.4$ & -9.6 & $41.5 \pm 1.4$ \\
\hline
\end{tabular}

* Sample diluted with inactive $\mathrm{CO}_{2}$.

Comment: low $\Delta$ value for collagen agrees with previous results (Harkness and Walton, 1972; Libby et al., 1964; Berger et al., 1966) and indicates slow turnover rate. The $\Delta$ value for heart, GU-438, is abnormally low relative to other heart values. Although variations exist within and between series, brain and muscle data are generally consistent and may reflect turnover rates faster than in other tissues.

\section{ATMOSPHERIC SAMPLES}

Monthly atmospheric samples from sampling stas. at Lerwick $\left(60^{\circ}\right.$ $08^{\prime} \mathrm{N}$ Lat, $01^{\circ} 11^{\prime} \mathrm{W}$ Long), Snowdon (53 $03^{\prime} \mathrm{N}$ Lat, $04^{\circ} 00^{\prime} \mathrm{W}$ Long) and at Chilton (51 $31^{\prime} \mathrm{N}$ Lat, $01^{\circ} 20^{\prime} \mathrm{W}$ Long) are being received at this lab. Although analysis of every sample is not possible at present, representative samples are being measured to supplement tissue data. $\mathrm{CO}_{2}$ is coll. by exposure of carbonate-free $8 \mathrm{~N} \mathrm{KOH}$ solution to the atmosphere for each calendar month. 
Lerwick series, 1970-1971

Samples coll. by Meteorologic Office in their ventilated East hut, Lerwick.

\begin{tabular}{lllccc}
\hline Sample no. & \multicolumn{2}{c}{ Coll. date } & $\delta \mathrm{C}^{14} \%$ & $\delta \mathrm{C}^{13 \%} \%$ & $\Delta \%$ \\
\hline GU-441 & April & 1970 & $53.0 \pm 1.3$ & -15.2 & $50.0 \pm 1.3$ \\
GU-442 & May & 1970 & $51.0 \pm 0.9$ & -15.4 & $48.1 \pm 0.9$ \\
GU-443 & June & 1970 & $45.9 \pm 1.3$ & -15.1 & $43.0 \pm 1.3$ \\
GU-444 & July & 1970 & $57.9 \pm 0.9$ & -15.1 & $54.8 \pm 0.9$ \\
GU-445 & Nov. & 1970 & $54.3 \pm 0.7$ & -17.6 & $52.0 \pm 0.8$ \\
GU-446 & March & 1971 & $53.0 \pm 0.9$ & -16.8 & $50.5 \pm 1.0$ \\
GU-447 & June & 1971 & $56.8 \pm 0.9$ & -18.9 & $54.9 \pm 0.9$ \\
\hline
\end{tabular}

Snowdon series, 1970-1971

$\mathrm{CO}_{2}$ coll. by Central Electricity Generating Board in a ventilated cabinet at Cwm Dyli Power Sta. on E slope of Mt. Snowdon.

\begin{tabular}{lllccc}
\hline Sample no. & \multicolumn{2}{c}{ Coll. date } & $\delta \mathrm{C}^{14 \%}$ & $\delta \mathrm{C}^{13 \%} \%$ & $\Delta \%$ \\
\hline GU-448 & May & 1970 & $48.0 \pm 0.9$ & -16.3 & $45.4 \pm 0.9$ \\
GU-449 & Nov. & 1970 & $57.5 \pm 0.9$ & -16.7 & $54.9 \pm 0.9$ \\
GU-450 & March & 1971 & $50.0 \pm 0.9$ & -17.4 & $47.7 \pm 0.9$ \\
GU-451 & June & 1971 & $51.8 \pm 0.9$ & -15.7 & $49.0 \pm 0.9$ \\
\hline
\end{tabular}

Comment: $\Delta$ values still show seasonal fluctuations with maximum activity from June to July. Average $\Delta$ value from April 1970 to March 1971 is ca. $50 \%$, thus during period most recent to coll. and measurements of tissue samples the average air $\Delta$ values are ca. $50 \%$.

REFERENCES

Baxter, M. S., Ergin, M., and Walton, A., 1969, Glasgow University radiocarbon measurements I: Radiocarbon, v. 11, p. 43-52.

Baxter, M. S. and Walton, A., 1970, Glasgow University radiocarbon measurements III: Radiocarbon, v. 12, p. 496-502.

1971, Fluctuations of atmospheric carbon-14 concentrations during the past century: Royal Soc. [London] Proc. A, v. 321, p. 105-127.

Berger, Rainer and Libby, W. F., 1966, UCLA radiocarbon dates V: Radiocarbon, v. 8 , p. $467-497$

Ergin, M., Harkness, D. D., and Walton, A., 1970, Glasgow University radiocarbon measurements II: Radiocarbon, v. 12, p. 486-495. 14 , p. $321-325$.

Harkness, D. D. and Walton, A., 1972, Glasgow University radiocarbon measurements IV: Radiocarbon, v. 14, p. 111-113.

I.ibby, W. F., et al., 1964, Replacement rates for human tissues from atmospheric radiocarbon: Science, v. 146, p. 1170-1172. 